\title{
Paroxysmal Nocturnal Hemoglobinuria
}

National Cancer Institute

\section{Source}

National Cancer Institute. Paroxysmal Nocturnal Hemoglobinuria. NCI Thesaurus. Code C61233.

A rare acquired hematologic disorder characterized by hemolytic anemia, dark-colored urine due to the release of hemoglobin in the blood, and thrombosis. The episodes of hemolysis tend to occur at night. It is caused by a somatic mutation in the glycosylphosphatidylinositol biosynthesis gene. 